\title{
I.ARTICLES
}

\author{
MONIKA JANICKA \\ Uniwersytet Marii Curie-Skłodowskiej w Lublinie \\ monika.janicka@poczta.umcs.lublin.pl \\ ORCID: 0000-0002-5603-4803
}

\section{Effizienz der Methode Lernen durch Lehren (LdL) - Ergebnisse eines Experiments}

\section{Efficiency of the learning through teaching (LTT) method - experimental results}

\begin{abstract}
Constructivist learning approaches emphasize the interface of learning by doing and student participation, as well as the efficiency of the learning process. Research findings have also demonstrated that students learn more effectively from each other (Hattie 2013). The aim of the experiment conducted was to verify the efficiency of the "learning through teaching" method (LTT) (Martin 1985), in which students assume some of the teacher's tasks. The experiment was conducted during the third semester of the "grammar in communication" course at the faculty of Applied Linguistics at Maria Curie-Skłodowska University. The result confirmed that, after a semester, the group working with the learning through teaching method had higher achievements than the control group. Thanks to a qualitative survey, some prospects and limits of the method could be determined. It also provided feedback for intern, process-oriented evaluation, which makes it possible to optimize course design in the future.
\end{abstract}

KEYWORDS: constructive didactics, learning through teaching (LTT), efficiency, experiment, prospects and limits.

SCHLÜSSELWORTE: konstruktivistische Didaktik, Lernen durch Lehren (LdL), Effizienz, Experiment, Möglichkeiten und Grenzen. 


\section{EINLEITUNG}

Der Paradigmenwechsel in der Didaktik, der die Lerner in den Mittelpunkt der Lehr-Lern-Prozesse stellt und diesen Prozess als einen konstruktiven Akt betrachtet, beeinflusste auch die Zugänge zur Fremdsprachendidaktik. Konstruktivistische Didaktiker betonen, dass sich das Wissen von einer Person auf die andere nicht übertragen lässt, weil Lernen eine autopoietische Tätigkeit des menschlichen Gehirns sei. Lernen sei zugleich Handeln, denn "by doing lernen wir" (Siebert 2005: 21). Je handlungsreicher das Lernen ist, als desto viabler, brauchbarer und funktionaler wird es von den Lernern wahrgenommen (Reich 2004: 161). Um dieser Erkenntnis gerecht zu werden, müssen Unterrichtsmethoden und -formen überlegt werden, die aktive Lernprozesse sowie aktive Teilnahme der Schüler am Unterricht ermöglichen. Da die Verantwortung für die Lernprozesse von der Lehrperson immer stärker in Richtung Lerner verschoben wird, steigt auch die Nachfrage nach der Partizipation Letzterer und nach deren Mitwirkung am Unterrichtsgeschehen. Reich betont, dass in der konstruktivistischen Didaktik alle Lerner auch Didaktiker seien, die eine hohe Erlebnisdichte und vor allem Antriebe benötigten, um ihr Lernen erfolgreich zu gestalten (Reich 2004: 59).

Ganz neu muss hinsichtlich dieses Paradigmenwechsels die Rolle des Lehrers gedacht werden. Angesichts der überall präsenten Digitalisierung und dem raschen Veralten des Wissens sind Unterrichtsmethoden, deren Ziel es ist, möglichst viel Wissen in möglichst kurzer Zeit zu vermitteln, zum Scheitern verurteilt. An Bedeutung gewinnen wiederum solche didaktischen Ansätze, die selbständiges Erkunden und Entdecken ermöglichen. Die in traditionellen Lehransätzen primäre Aufgabe der Lehrpersonen, das Wissen zu vermitteln, verliert an Relevanz. In den Vordergrund tritt dafür ihre Rolle als Pädagogin, die für die Organisation, Steuerung und Kontrolle der Lernprozesse zuständig ist (Pfeiffer 2004: 80-81). In konstruktivistischen Lernumgebungen sollen Lehrpersonen eine Atmosphäre herstellen und gewährleisten, in der optimal gelernt werden kann. Dazu gehören die Maximierung von aktiver Lernzeit, in der sich die Lerner konstruktiv und engagiert mit dem jeweiligen Gegenstand beschäftigen, Aufstellen klarer Regeln und das Einfordern ihrer Einhaltung sowie die Planung und Vorstrukturierung von Unterrichtsabläufen (Gold 2015: 113, nachfolgend Helmke 2014).

Die Grundsätze der konstruktivistischen Didaktik und Pädagogik wiederspiegeln sich in der von Jean-Pol Martin entwickelten Unterrichtsmethode. Aus Kritik an den in den 80er Jahren des 20. Jahrhunderts den Fremdsprachenunterricht prägenden behavioristischen Lerntheorien, die lediglich 
auf Repetition und Imitation beruhten und den Lehrer als Vorbild in den Mittelpunkt der Lernprozesse stellten und durch Hinwendung zu dem von Piepho (1974) entwickelten kommunikativen Ansatz, dessen Ziel es war, die Sprechtätigkeit der Schüler anzuregen und deren Sprechanteil zu vergrößern sowie zum Sprechen zu motivieren, entwickelte und popularisierte Martin eine Methode, die das Handeln der Schüler stark in den Vordergrund rückt und ihnen größere Partizipation und Verantwortung am Unterricht ermöglicht. Die Methode steht unter starkem Einfluss der kognitiven Lerntheorien und setzt voraus, dass das Lernen ein konstruktiver Akt der Informationsverarbeitung sei. Je stärker man sich mit dem Gegenstand, im Falle des Erlernens einer Fremdsprache - mit dem „unfiltrierten sprachlichen Angebot" - auseinandersetze, desto größer sei die Chance, dass dieser im Gedächtnis behalten bleibe (Martin 1997: 3-4).

\section{LERNEN DURCH LEHREN - BESCHREIBUNG DER METHODE}

Als Praktiker versuchte Martin die Prämissen der kognitiven Lerntheorien (die Lerner setzen sich mit dem Stoff kognitiv auseinander), des kommunikativen Ansatzes (sie kommunizieren miteinander) und des behavioristisch geprägten Zugang zu den Lernprozessen (die Lerner habitualisieren die erlernten Strukturen dadurch, dass sie sie wiederholen) zu integrieren. Diese Integration sei aufgrund des Informationsverarbeitungsansatzes möglich, der auf folgendem Zyklus basiert:

- Informationsinteresse,

- Informationsaufnahme,

- Informationsverarbeitung,

- Informationsspeicherung,

- Reaktivierung der gespeicherten Information,

- Informationsanwendung.

Für Informationsinteresse, -verarbeitung und -anwendung kann gesorgt werden, indem die Lerner die zu lernenden Inhalte mit Hilfe der Lehrperson aufbereiten und ihren Mitschülerinnen und Mitschülern vermitteln. Dies setzt wiederum voraus, dass die entsprechenden linguistischen und didaktischen Kompetenzen der Lernenden aufgebaut werden, wofür die Lehrperson zuständig ist (Martin 1986: 398-399).

Als Ausgangspunkt zur Entwicklung der LdL-Methode diente die Beobachtung, dass die Lernenden lediglich durch $25 \%$ des Unterrichts sprachlich aktiv sind. Dank des Tauschs der traditionellen Lehrer-Schüler-Rollen, indem die Lerner einen Teil der traditionellen Lehreraufgaben wie Korrek- 
tur der Hausaufgaben oder Vorlesen eines Diktattextes übernehmen, gelang es, den bisher knappen Redeanteil der Lerner um das Dreifache zu steigern (Martin 1985: 216ff).

Damit aktive Beteiligung der Lerner möglich wird, müssen bestimmte Regeln eingehalten werden:

- Der Stoff muss zu Hause vor- und nachbereitet werden, sonst wären die Lernenden nicht in der Lage, sich am Unterricht aktiv zu beteiligen,

- Der Klassendiskurs muss darauf ausgerichtet sein, dass die Lernenden einander konzentriert zuhören und Fragen stellen, wenn etwas in der Darstellung ihrer Mitschüler nicht klar ist,

- Die Lernenden, die den Unterricht leiten, stellen das Thema vor und lassen ihre Mitschüler überlegen, was sie bereits zu dem zu behandelnden Thema wissen,

- Die den Unterricht leitenden Lernenden führen das Thema in kleinen Portionen ein mit der ständigen Rückfrage, ob der Stoff von den Mitschülern verstanden wurde,

- Im Anschluss erfolgt die zweite Vertiefung des Stoffes, indem die Lernenden die erlernten Strukturen aktiv, z.B. als Rollenspiel oder symbolisch, z.B. als Standbild oder Zeichnung anwenden (Martin \& Oebel 2007: 9).

Bei diesen Vorgängen muss eine Reihe "konstruktiv-kommunikativer Reflexe“ bei den Lernenden hervorgerufen werden:

- Sie müssen daran gewöhnt werden, für Fehlerkorrekturen passable grammatische Erklärungen liefern zu können,

- Sie müssen dazu angeleitet werden, unklare Stellen aufzugreifen und den Klassenraumdiskurs darauf bezogen anzuleiten,

- Sie müssen daran gewöhnt werden, die Lernenden aus der Gruppe anzusprechen, die über Spezialwissen verfügen und dieses Wissen entsprechend nutzen, um das Wissensniveau der gesamten Gruppe zu erhöhen,

- Sie müssen in der Lage sein (spontan) Visualisierungen des vermittelten Stoffes vorzunehmen, die zum Verständnis beitragen können,

- Sie müssen daran gewöhnt werden, durch kleine Rollenspiele abstrakte Situationen nachvollziehbar zu machen (Martin \& Oebel 2007: 8).

Einschlägige Untersuchungen, die punktuell einige Unterrichtsprinzipien der LdL-Methode aufgreifen, bestätigen die Effizienz dieser Unterrichtshandlungen, die aktive Beteiligung der Lerner und ihre Eigenverantwortung ermöglichen. So haben Unterrichtsmethoden die auf Diskussionen in der Klasse beruhen und kooperatives Lernen voraussetzen, entsprechend mit dem Effekt von $d=0.82$ und $d=0.59$ einen beträchtlichen Einfluss auf die 
Effizienz der Lernprozesse (Hattie 2015). Trotz wissenschaftlicher Evidenz (der Einfluss von $d=0.54$ ), dass die Schüler effektiver Lernen, wenn sie ihre eigenen Lehrer sind, zeigen z.B. die Untersuchungen von Yair (2000), dass die Lehrerinnen und Lehrer etwa 70-80\% der gesamten Unterrichtszeit monologisch an sich reißen ${ }^{1}$. Einen signifikanten Einfluss auf die Effizienz der Lernprozesse weisen Handlungen der Lehrpersonen nach, in denen sie sich als Förderer der Lehr-Lern-Prozesse wahrnehmen und den Lernern durch fördernde Bewertung Impulse zum Weiterlernen und zur Reflexion über den Lernprozess liefern $(d=0.90)$ (Hattie 2015).

\section{ZIELSETZUNG UND BESCHREIBUNG DES EXPERIMENTS}

Untersuchungen, die explizit die Effizienz der LdL-Methode dokumentieren, sind eher dünn gesät. Deshalb war das Ziel der durchgeführten Untersuchung, einen weiteren Nachweis für die Effizienz der LdL-Methode zu liefern, und zwar im universitären Kontext. Als Forschungsmethode wurde das Experiment gewählt, weil es die „Überprüfung von bereits theoretisch festgelegten Aussagen nach festgelegten Bedingungen“ ermöglicht (Atteslander 2003: 196).

Der Kurs "Grammatik in Kommunikation", in dem das Experiment durchgeführt wurde, wurde mit Studenten der Angewandten Linguistik an der Maria Curie-Skłodowska in Lublin durchgeführt und umfasste 30 Unterrichtseinheiten à 45 Minuten. Die Beteiligten waren Studenten des dritten Semesters, die Englisch als erste und Deutsch als zweite Fremdsprache studierten. $^{2}$ Das durchgeführte Experiment war ein Feldexperiment, in dem „zwei kontrastierende Gruppen in ihrer realen Umwelt untersucht werden können, von denen eine dem (angenommenen) Kausalfaktor [= unabhängige Variable, in diesem Fall dem Unterrichten nach Prinzipien der LdL-Methode] ausgesetzt" war (Atteslander 2003: 200).

Die Experimentalgruppe bestand aus 12 Personen, deren Notendurchschnitt am Ende des zweiten Semesters 3,4 betrug. Die Kontrollgruppe bestand aus 13 Personen mit einem Notendurchschnitt von 3,53. In der Experimental-

\footnotetext{
${ }^{1}$ Dies bestätigen auch die bereits erwähnten Untersuchungen von Martin (1985).

2 Der Kurs „Grammatik in Kommunikation“ umfasst für diese Sprachenkombination drei Semester. Das Experiment wurde in dem letzten Kurssemester durchgeführt.

${ }^{3}$ Das Notensystem an polnischen Universitäten hat sechs Stufen: 5 ist die höchste Note und entspricht der Beschreibung "sehr gut" , 4 = gut, 3 = befriedigend. 2 ist die niedrigste Note und gilt als "ungenügend". Darüber hinaus gibt es zwei Zwischennoten: gut plus $(4,5)$ und befriedigend plus $(3,5)$.
} 
gruppe übernahmen die Studierenden die Unterrichtsführung, während in der Kontrollgruppe die gleichen Themen traditionell von der Lehrperson vermittelt wurden. Am Anfang des Semesters wurden die Studenten mit der LdL-Methode vertraut gemacht: ihnen wurden eine Power-Point-Präsentation und ein Film zu den Grundsätzen der LdL-Methode gezeigt. In dem Kurzfilm (zugänglich unter dem Link https://www.youtube.com/watch?v=wyj JMjBodtQ) wurden auch Vorteile der Methode aufgelistet. Als Nächstes habe ich den Studierenden meine Erwartungen hinsichtlich des Forschungsprojekts vorgestellt. Die Studierenden teilten sich in Paare bzw. Dreiergruppen auf, in denen sie die durchzuführenden Unterrichtssequenzen bearbeiten sollten. Das für das Semester zum Einstudieren vorgesehene Thema (Syntax der deutschen Sprache) wurde in kleinere, thematische Einheiten aufgeteilt und an die Paare bzw. Kleingruppen verteilt, wobei jede Gruppe z.T. die Wahl hatte, mit welcher Struktur sie sich ausführlich befassen möchte. Auch die Präsentationstermine wurden am Anfang des Semesters festgelegt. Die erste Gruppe hatte drei Wochen, um ihren Stoff aufzuarbeiten. Die Studierenden wurden dazu verpflichtet, während der Vorbereitungszeit an Sprechstunden teilzunehmen und den von ihnen bearbeiteten Stoff sowie den geplanten Unterrichtsablauf zu konsultieren. Darüber hinaus wurden sie beauftragt, Listen mit voraussichtlich nötigen Wendungen, der sog. Unterrichtssprache, vorzubereiten und sie zur sprachlichen Kontrolle vorzulegen. Das hat sie dazu veranlasst, die Unterrichtssprache einzuüben und den während ihrer Präsentation benötigten Wortschatz parat zu haben.

Das Experiment sollte eine quantitative Antwort auf die Forschungsfrage liefern:

1. Inwieweit erhöht die LdL-Methode die Effizienz der Lehr-Lern-Prozesse im Kurs "Grammatik in Kommunikation“?

Die von der Experimentalgruppe direkt im Anschluss an die Prüfung in deutscher Grammatik ausgefüllten Fragebögen sollten dazu verhelfen, die Wahrnehmung der LdL-Methode aus der Innenperspektive der Beteiligten zu erforschen. Die zu beantworteten Forschungsfragen lauteten:

2. Wie beurteilen die Studierenden das Lernen mithilfe der LdL-Methode?

2.1. Welche Kompetenzen haben sie dank der LdL-Methode erworben?

2.2. Auf welche Schwierigkeiten sind sie dabei gestoßen?

3. Wie bewerten die Studierenden die Unterstützung seitens des Hochschullehrers / des Kursleiters? Wo sehen sie weiteren Förderbedarf?

Die Inhaltsanalyse der Fragebögen sollte auch Impulse liefern, die Unterrichtsplanung nach den LdL-Prinzipien in Zukunft lernfreundlicher und effizienter zu gestalten. 


\section{BESCHREIBUNG DER FORSCHUNGSINSTRUMENTE UND DER ERGEBNISSE DES EXPERIMENTS}

Wie das Ergebnis der Grammatikprüfung, die am Ende des dritten Semesters durchgeführt wurde, zeigt, erreichte die Experimentalgruppe, die über ein ganzes Semester hinweg nach den Prinzipien der LdL-Methode in das Unterrichten involviert war, die Durchschnittsnote von 3,3, während die traditionell von der Lehrperson unterrichtete Kontrollgruppe den Durchschnitt von lediglich 2,9 erreichte. Das Experiment wurde zwar in einer kleinen Gruppe durchgeführt und vermag keine Allgemeingültigkeit zu beanspruchen, sein Ergebnis lässt aber die Schlussfolgerung formulieren, dass sich eine stärkere Beteiligung der Studierenden am Unterrichtsgeschehen positiv auf den Lerneffekt auswirkt.

Die subjektiven Urteile der Studierenden hinsichtlich der LdL-Methode (Forschungsfrage 2), waren überwiegend positiv, was das Diagramm 1 schildert. Selbst wenn $42 \%$ der Beteiligten die Arbeit nach Prinzipien der LdL-Methode als „mittelmäßig“ einschätzten, beurteilte niemand diese Methode eindeutig negativ.

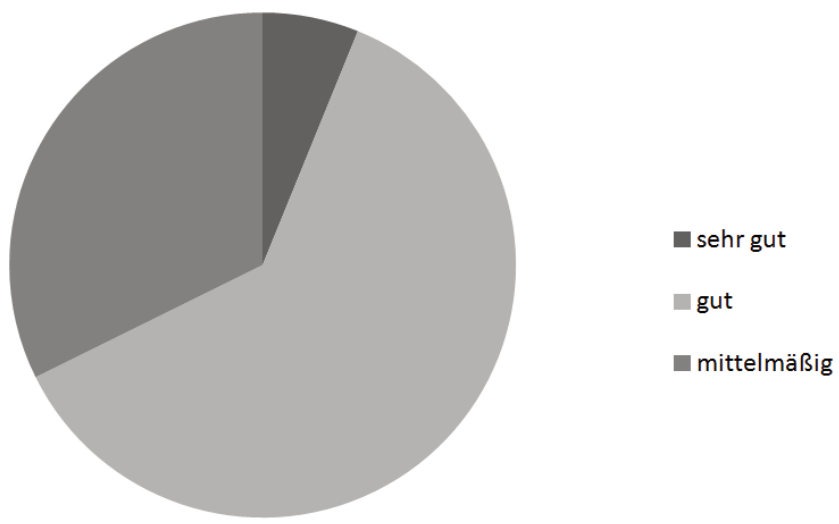

Diagramm 1. Anwendung der LdL-Methode im Kurs "Grammatik in Kommunikation" nach subjektiver Einschätzung der Beteiligten

Um die Vorteile sowie die dank der LdL-Methode erworbenen Kompetenzen einschätzen zu können wurde eine Antwortliste mit 12 Items vorgeschlagen. Sie umfassten:

- sprachlich-kognitive Kompetenzen: Ich wurde zum Kommunizieren in der Fremdsprache gezwungen. / Ich hatte mehr Zeit zum Kommunizieren in der Fremdsprache. / Ich habe besser den grammatischen 
Stoff verstanden. / Ich habe meine Sprachkompetenz in Deutsch verbessert;

- affektive Kompetenzen: Ich war motivierter / Ich habe mehr Selbstwertgefühl gewonnen. / Ich habe meine Sprechhemmungen überwunden. / Ich war aktiver als während des traditionellen Unterrichts;

- strategisch-methodische Kompetenzen: Ich habe besser verstanden, wie ich lernen soll / Ich habe gelernt, die Aufmerksamkeit der Gruppe zu kontrollieren / Ich habe verstanden, worauf ich beim Unterrichten achten soll / Ich kann davon während meines Praktikums an Schulen profitieren. ${ }^{4}$

Zu den drei am häufigsten erwähnten Vorteilen der LdL-Methode zählte der Erwerb von sprachlich-kognitiven Kompetenzen:

Ich wurde zum Kommunizieren in der Fremdsprache gezwungen (6 Personen). Ich war aktiver als während des traditionellen Unterrichts (5 Personen).

Ich hatte mehr Zeit für das Sprechen in der Fremdsprache (4 Personen).

$\mathrm{Zu}$ den drei am häufigsten genannten Schwierigkeiten, die die befragten Personen hinsichtlich des LdL-Unterrichts zum Ausdruck brachten, gehörten:

1. großer Stress (8 Personen),

2. Mangel an sprachlicher Vorbereitung (8 Personen),

3. geringere Qualität des Unterrichts, als wenn er von einer erfahrener Fachkraft durchgeführt worden wäre (7 Personen).

Hinsichtlich des ersten Faktors konnte tatsächlich beobachtet werden, dass es für die meisten Unterrichtenden eine emotionale Herausforderung war, vor der Gruppe aufzutreten, in die Rolle eines Experten zu schlüpfen, das Unterrichtsgeschehen zu leiten und ständig unter Kontrolle zu haben. Trotz der Stressempfindung konnte jedoch einzelnen Aussagen entnommen werden, dass die Zusammenarbeit mit der Gruppe viel Spaß bereitete und immer mit einem netten Akzent [z.B. in Form von Spielen oder anderen kreativen Aktivitäten - Ergänzung M.J.] endete ( $\mathrm{Fb} 3)$. Bei einigen dürfte der Stress aus unzureichender Vorbereitung resultieren ( $\mathrm{Fb} 6)$. Tatsächlich konnten nur wenige Beteiligte ihre Unterrichtssequenz reibungslos und aufgelockert durchführen. Aber alle bereicherten den Unterricht mit kreativen Ideen zum Einüben von Strukturen bzw. zur sprachlichen Produktion. Das Projekt hatte die Erweiterung der sprachlichen Kompetenz zum Ziel. Einige Beteiligte konnten ihre sprachlichen Defizite erst während der Unterrichtsführung feststellen, was bei ihnen zu einer gewissen Frustration, im nächsten

${ }^{4}$ Die überwältigende Mehrheit der Gruppe (9 Personen) hat Englisch als Fremdsprache auf das Lehramt studiert. Dieser Teil der Gruppe hatte 120 Stunden Praktikum an Schulen während des Bachelorstudiums zu absolvieren. 
Schritt aber zur Reflexion über die eigenen Stärken und Schwächen führte. Denn zugleich erschloss sich dank Unterstützung der Lehrperson die Möglichkeit, die nicht im eigenen Vokabelschatz vorhandenen Strukturen kennenzulernen und sofort anzuwenden. 75\% der Beteiligten schätzten die Unterstützung durch die Lehrperson sowohl in der Vorbereitungsphase als auch während der Präsentation als "sehr gut" (75\%) bzw. als "gut“ (25\%) ein.

Die Frage nach der Rolle des Fachlehrers (Forschungsfrage 3) war eine offene Frage. Deshalb konnten die erhobenen Daten einer qualitativen Inhaltsanalyse unterzogen werden (vgl. Mayring 2002: 107-109). Zwei Aspekte stechen aus diesen Aussagen heraus. Die meisten Beteiligten betonten die stetige fachliche und methodische Unterstützung seitens der Lehrperson bei Missverständnissen, Schwierigkeiten oder Fehlern. Mit dieser Unterstützung konnten sie sowohl vor, als auch während der Präsentation rechnen: ${ }^{5}$

Der Lehrer hat während der Sprechstunde die Fehler in den Aufgabenblättern korrigiert, hat Aufgaben vorgeschlagen und [die Studierenden] beim Zeitmanagement während des Unterrichts unterstützt (Fb 10). ${ }^{6}$

Man konnte früher in die Sprechstunde kommen, um den geplanten Unterricht zu besprechen, die Fehler in den Handouts zu korrigieren. Während des Unterrichts erklärte der Lehrer komplexere Aspekte ( $\mathrm{Fb} 5)^{7}$

Von Wichtigkeit war für die Studierenden auch die Zugänglichkeit der Lehrperson:

Man konnte den Fachlehrer früher um Rat fragen, per E-Mail und dann in der Sprechstunde, in der der Lehrer ausführlich die von Studenten vorbereiteten Materialien besprach und wertvolle Hinweise gab (Fb 9). ${ }^{8}$

Zum Schluss wurden die Befragten gebeten, ihren persönlichen Kommentar zu dem nach der LdL-Methode durchgeführtem Grammatikkurs abzugeben. Viele Kommentare waren positiv. Darin kam zwar der große Arbeitsaufwand, den die Studierenden bei der Vorbereitung und Durchführung des Unterrichts zu leisten hatten, öfters zur Sprache. Die Beteiligten sahen aber zugleich die Vorteile ihrer Anstrengungen. Besonders diejenigen,

${ }^{5}$ Alle zitierten Aussagen wurden von Monika Janicka übersetzt.

${ }^{6}$ "Nauczyciel podczas konsultacji poprawił błędy w przygotowanych materiałach, zaproponował zadania i pomógł w organizacji czasu na zajęciach".

7 „Można było wcześniej przyjść na konsultacje, aby omówić lekcje i poprawić błędy w materiałach, podczas lekcji nauczyciel wyjaśniał bardziej skomplikowane rzeczy".

8 ,Z nauczycielem przedmiotu można było skonsultować się wcześniej poprzez e-mail, a następnie na konsultacjach, gdzie nauczyciel dokładnie omówił przygotowane przez studenta materiały i dał cenne wskazówki". 
die auf das Lehramt studierten, betrachteten es als eine wichtige didaktische Erfahrung:

Ich finde, dass der diesjährige Kurs eine interessante Idee war, weil jeder Studierende die Chance hatte, seine Kompetenzen unter Beweis zu stellen, sich in der Lehrerrolle zu bewähren. Das hat jeden viel Mühe gekostet. Durch verschiedene Spiele oder Aufgaben am Ende des Unterrichts konnten wir den neuen Stoff festigen und produktiv anwenden $(\mathrm{Fb} 9) .{ }^{9}$

Der Kurs war eine interessante Erfahrung, sowohl für die Person, die sich in die Lehrerrolle versetzte, als auch für die Teilnehmenden. Der Kurs hat eine wichtige Rolle gespielt und ermöglichte den Personen, die sich auf die Schulpraktika vorbereiten, Erfahrungen zu sammeln. Es ermöglichte seine Stärken und Schwächen, an denen man arbeiten soll, kennenzulernen. ( $\mathrm{Fb} 7){ }^{10}$

Es gab jedoch auch kritische Stimmen. Sie bezogen sich vor allem auf die angenommenen fachlichen und sprachlichen Defizite anderer Studierender:

(...) manche Themen waren für die Präsentierenden unverständlich und sie wiederholten nur die Regeln (Fb 8). ${ }^{11}$

Manchmal machten die Unterrichtenden Fehler in dem präsentierten Thema oder sie kannten die Antworten zu den Aufgaben gar nicht. [Ich würde mir wünschen], dass die Studenten besser auf die Durchführung des Unterrichts vorbereitet wären (...) [und dass sie] den Stoff besser erklären könnten $(\mathrm{Fb} 6){ }^{12}$

Ich finde, dass die Qualität des Unterrichts, dadurch dass er nicht von der Lehrperson durchgeführt worden war, gesunken ist $(\mathrm{Fb} 3) .{ }^{13}$

An dieser Stelle sollte jedoch erklärend noch einmal darauf hingewiesen werden, dass die Prüfungsergebnisse, die den Studierenden zum Zeitpunkt

9 „Uważam, że tegoroczny kurs był ciekawym pomysłem, ponieważ każdy student mógł się wykazać, sprawdzić się w roli nauczyciela, wymagało to od każdego sporo wysiłku. Poprzez różne gry czy zadania na koniec prowadzonej lekcji mogliśmy zastosować i utrwalić nowy materiał w mówieniu".

10 „Kurs był ciekawym doświadczeniem zarówno dla osoby odgrywającej rolę nauczyciela, jak i osoby uczestniczącej. Kurs ten odgrywał ważną rolę i pozwalał zdobyć doświadczenie osobom, które odbywają praktyki metodyczne. Pozwoliło to zaznajomić się ze swoimi mocnymi, ale także słabymi stronami, nad którymi należy pracować".

11, „...) niektóre tematy były niezrozumiałe dla prowadzących i polegali tylko na regułkach".

12 [Życzyłabym sobie] „aby studenci prowadzący lekcję byli bardziej przygotowani do prowadzenia zajęć (...) i potrafili lepiej wytłumaczyć temat prowadzonych zajęć w języku niemieckim. (...) Czasem studenci prowadzący lekcje popełniali błędy w prezentowanym temacie lub sami nie znali odpowiedzi na zadania".

13 „Sądzę, że jakość zajęć pogorszyła się przez to, że nie były one prowadzone przez nauczyciela". 
der Ausfüllung des Fragebogens nicht bekannt waren, dokumentieren, dass sich die angeblich schlechtere Qualität des Unterrichts auf die Leistungen der Studierenden auf keinen Fall negativ ausgewirkte. Die Ergebnisse der Prüfung zeigen das Gegenteil. Es ist zuzugeben, dass die Studierenden unterschiedlich auf die Durchführung ihrer Aufgabe vorbereitet waren. Das dürfte nicht unbedingt an mangelnder Fachkompetenz, sondern eher an fehlendem Selbstvertrauen, an der Herausforderung vor der Gruppe zu stehen und Verantwortung für das Unterrichtsgeschehen zu übernehmen, liegen. Das war selbst für diejenigen Studierenden, die auf das Lehramt studierten, eine emotional belastende Erfahrung, was in den Fragebögen wiederholt zur Sprache kam.

Sehr wertvoll für die Auswertung des Experiments waren auch Verbesserungsvorschläge und Wünsche, die von einigen Studierenden formuliert wurden. Sie kamen jedoch leider selten vor. Geäußert wurde das Bedürfnis, den gesamten Stoff, der im Laufe des Semesters durchgenommen wurde, gegen Ende Januar vor der Prüfung zu wiederholen oder die Qualität der Handouts vor allem durch Visualisierungen zu verbessern. Bei der Beantwortung dieser Frage wurde auch der Wunsch zum Ausdruck gebracht, die Lehrperson solle den Studierenden bereits bearbeitete Hilfsmaterialien mit den wichtigsten Strukturen zur Verfügung stellen. Das würde nach Annahme der befragten Person die Qualität der Materialien sichern. Einer der Wünsche war komplexer:

Wir sollten gemeinsam, (z.B. mit der ganzen Gruppe) ein Musterunterrichtsszenario ausarbeiten, nützliche Wendungen, Unterrichtssprache (Aufforderungen, Bitten), die eine zuverlässige Basis, auf die sich Studierende stützen könnten, bilden würden. Das würde den Unterrichtenden mehr Selbstvertrauen geben $(\mathrm{Fb} 11) \cdot{ }^{14}$

Weitere Veränderungsvorschläge betrafen den Wunsch nach Visualisierungen, die den Stoff durch Tabellen, Schemata und grafische Hervorhebungen ergänzen würden. Geäußert wurde auch das Bedürfnis nach einer Überprüfung der Stoffaneignung in Form eines Quiz. Es gab auch eine Stimme, dass die Übungen abwechslungsreicher gestaltet werden sollten.

Diese Hinweise werden möglichst weitgehend in den nachfolgenden Kursgruppen berücksichtigt, zumal sie zur Optimierung der Unterrichtsabläufe beitragen können.

14 [Życzyłabym sobie, aby], „opracować wspólnie (np. razem z całą grupą) przykładowy scenariusz lekcji, przydatne zwroty, wyrażenia związane z prowadzeniem lekcji (polecenia, prośby), które byłyby rzetelną bazą, na której studenci mogliby się opierać. Myślę, że zaowocowałoby to większą pewnością siebie osób prowadzących zajęcia". 


\section{SCHLUSSFOLGERUNGEN UND DISKUSSION}

Zwar waren fast 60 Prozent der Befragten davon überzeugt, dass der von den Studierenden geleitete Unterricht von geringerer Qualität und Effizienz als der von erfahrener Lehrperson durchgeführte Unterricht sei, aber die Prüfungsergebnisse haben diese Befürchtung nicht bestätigt. Ganz im Gegenteil: Aufgrund des Vergleiches der Leistungsergebnisse in der Experimental- und der Kontrollgruppe lässt sich schlussfolgern, dass sich die Partizipation, Engagement und Mitwirkung von Studierenden fördernde LdL-Methode effektiver auf ihre Leistungen auswirkt als die traditionelle Unterweisung durch die Lehrperson. Die Ergebnisse des durchgeführten Experiments wurden deshalb im Jahr darauf der nachfolgenden Gruppe vorgestellt, um die Studierenden von Arbeit mit der LdL-Methode zu überzeugen und ihnen Angst vor Misserfolg vorweg zu nehmen.

Das Experiment zeigte deutlich, dass das LdL-Konzept stark veränderte Aufgaben der Lehrperson impliziert. Sie wird vor allem zum Organisator der Lehr-Lern-Prozesse, zum Berater und Coach. Die Lehrperson unterstützt die Lernenden bei der Planung der Arbeit, Wahl der Aufgaben sowie beim Interagieren in Gruppen. Sie muss den Lernenden auch methodische Kompetenzen vermitteln, zumal die Studierenden entweder geringe oder keine Lehrerfahrungen hatten. Es entlastet sie allerding nicht von ihren Aufgaben als Experte, denn sie muss umso in dem zu vermittelndem Stoff bewandert sein, als sie den Unterricht im Voraus planen muss. Die Rolle der Lehrperson besteht hier schon am Anfang darin, den zu beherrschenden Stoff strukturiert aufzuteilen und danach die Teile wieder in ein komplexes Ganzes zusammenzufügen. Sie organisiert die Unterrichtsvorgänge, berät, unterstützt, kontrolliert und gibt Rückmeldungen und zwar nicht nur während des Unterrichts, sondern bereits lange davor. Unumgänglich ist deshalb ein gutes Zeitmanagement, eine sinnvolle Planung von Beratungsphasen, die den Lernenden genügend Zeit und Raum bieten, die Fachinhalte sowie das methodische Konzept des Unterrichts vorzustellen, zu besprechen und $\mathrm{zu}$ verbessern. Für die Lehrperson hat die Rolle des Coach und Prozessbegleiters den Vorteil, dass sie die Lernprozesse distanzierter beobachten und den künftigen Unterricht besser planen kann. Die gewonnenen Daten lieferten Feedback zur internen prozessbezogenen Evaluation (Gold 2015 nachfolgend Schmidt \& Perels 2010), auf deren Grundlage die unterrichtlichen Abläufe und Vorgehensweisen bei der künftigen Kursplanung optimiert werden können.

Die Forschungsergebnisse tun noch ein weiteres interessantes Forschungsfeld auf: Die Datenanalyse zeigte, dass die Beteiligten den Stressfaktor zu den größten Hürden bei der Arbeit nach der LdL-Methode zählten. 
Interessant wäre somit die dafür zuständigen Stressoren genauer zu eruieren und ggf. Strategien zu erkunden, die die Stressreaktionen zu reduzieren vermögen.

\section{LITERATURVERZEICHNIS}

Atteslander, R. (2003). Methoden der empirischen Sozialforschung. Berlin, New York: Walter de Gruyter.

Gold, A. (2015). Guter Unterricht. Was wir wirklich darüber wissen. Göttingen: Vandenhoeck \& Ruprecht.

Hattie, J. (2015). Widoczne uczenie się dla nauczycieli. Jak maksymalizować sitę oddziatywania na uczenie się. Warszawa: Centrum Edukacji Obywatelskiej.

Helmke, A. (2014). Unterrichtsqualität und Lehrerprofessionalität. Diagnose, Evaluation und Verbesserung des Unterrichts. Seelze: Kallmeyer.

Martin, J.-P. (1985). Zum Aufbau didaktischer Teilkompetenzen beim Schuler: Fremdsprachenunterricht auf der lerntheoretischen Basis des Informationsverarbeitungsansatzes. Tübingen: Narr.

Martin, J.-P. (1986). Für eine Übernahme von Lehrfunktionen durch Schüler. Praxis des neusprachlichen Unterrichts 4 (86), 395-403. http://www.ldl.de/Material/Publikationen/ lehrfunk.pdf (Zugriff am: 17.02.2019).

Martin, J.-P. (1997). Das Projekt "Lernen durch Lehren" - fachdidaktische Forschung im Spannungsfeld von Theorie und Praxis. http://www.ldl.de/LDL_ALT/material/aufsatz/ichenhau.pdf (Zugriff am: 17.02.2019).

Martin, J.-P. / Oebel, G. (2007). Lernen durch Lehren: Paradigmenwechsel in der Didaktik? Deutschunterricht in Japan. Heft 12, 4-21. http:/ / www.ldl.de/Material /Publikationen/ldl_ in_japan_paradigmenwechsel.pdf (Zugriff am: 17.02.2019).

Mayring, Ph. (2002). Einführung in die qualitative Sozialforschung. Weinheim und Basel: Beltz.

Pfeiffer, W. (2004). Interkulturowa glottopedagogika - nowa dyscyplina naukowa? Uwagi do dyskusji. In: K. Badstübner-Kizik / R. Rozalowska-Żądło / A. Uniszewska (Hrsg.), Sprachen lehren. Sprachen lernen. Nauczanie i uczenie się języków obcych (S. 71-84). Gdańsk: Wydawnictwo Uniwersytetu Gdańskiego.

Reich, K. (2004). Konstruktivistische Didaktik. Lehrern Und Lernen aus interaktionistischer Sicht. Berlin: Luchterhand.

Schmidt, M. / Perels, F. (2010). Der optimale Unterricht? Praxishandbuch Evaluation. Göttingen: Vandenhoeck \& Ruprecht.

Siebert, H. (2005). Pädagogischer Konstruktivismus. Lernerzentrierte Pädagogik in Schule und Erwachsenenbildung. Weinheim und Basel: Beltz.

Yair, G. (2000). Educational battlefields in America: The tug-of-war over students` engagement with instruction. Sociology of Education, 73 (4), 247-269.

Received: 28.08.2018; revised: 27.02.2019 